\title{
Mesoscopic modelling of conducting and semiconducting polymers
}

\author{
A M Stoneham ${ }^{1}$ and Marta M D Ramos ${ }^{2}$ \\ ${ }^{1}$ Centre for Materials Research, Department of Physics and Astronomy, \\ University College London, Gower Street, London WC1E 6BT, UK \\ 2 Departamento de Fisica, Universidade do Minho, Braga 4710-057, Portugal
}

Received 11 October 2000

\begin{abstract}
We present generalized Monte Carlo calculations to assess the effects of texture and related key factors on the properties of polymer-based light emitting diodes. We describe one class of mesoscopic model giving specific realizations of the polymer network. The model, with simple physically based rules, shows the effects of polymer structural order on current flow, trapping and radiative and non-radiative charge recombination within the polymer layer. Interactions between charges are included explicitly, as are image interactions with the electrodes. It is important that these Coulomb interactions are not simplified to an averaged space charge, since the local interactions can lead to effective trapping of charge, even in the absence of defective chains or impurity trapping. There proves to be an important role for trapping, in which charges are localized for times long compared with transit times. The competition between current flow, trapping and radiative and non-radiative charge recombination means that some of the trends are not intuitively obvious. For example, if radiative recombination occurs only on short polymer chains, as is the case for certain polymer systems, the internal efficiency appears to saturate for a concentration of these shorter luminescent chains of about $20-30 \%$. As the proportion of shorter chains increases, trapping increases, whereas current efficiency decreases. Our approach provides a natural link between atomistic models of individual polymer molecules and the macroscopic descriptions of device modelling. Such mesoscopic models provide a means to design better film structures, and hence to optimize the effectiveness of new organic materials in a range of applications.
\end{abstract}

\section{Introduction}

Conducting polymers, such as polyacetylene, are members of a novel class of materials. Some of these polymers mimic semiconductors, for example, by being the key components in lightemitting diodes (Burroughes et al 1990, Friend et al 1999). Even laser action has been claimed. Other classes of organics are metals, with a remarkably high conductivity for their weight. How can one model devices based on these polymers? Does it make sense to regard a thin film 
of polymer in the same way as a slab of conventional semiconductor of the same dimensions, bandgap and carrier mobility? We discuss the physics of these polymers, of which transpolyacetylene ( $\mathrm{t}-\mathrm{PA}$ ) is perhaps the best known example. Our emphasis is on how they can be modelled for device applications. This means that one should recognize the special types of electronic excitation, the large electron-phonon coupling and the texture, all of which point to a need for approaches different from those for conventional semiconductors. Our emphasis in this paper is on the modelling of the texture: how one can examine the effects of the range of chain lengths and configurations, and the general 'spaghetti structure' of the system.

The mesoscopic model we shall describe enables links to be made between the electronic and atomistic descriptions, which we and others have given elsewhere, and the more standard continuum device modelling. We shall need to discuss all three length scales. Certain of the features should carry over straightforwardly from a mesoscopic model to a continuum description. Space charge and the effects of trapped charges will certainly be a common feature for all length scales. There will surely be a Drude-like behaviour in the metallic limit. In some cases, there may be variable-range hopping. However, one cannot assume isotropy of carrier mobilities. Nor should one assume carrier injection is uniform over the electrode. At the other extreme, the intra-molecular properties of the polymer molecules will carry over to a mesoscopic model. Electron affinities, ionization potentials and carrier mobilities should be very similar in the solid state, at least after allowance has been made for the actual molecular configuration and for basic inter-molecular interactions. There will certainly be some new features: inter-molecular recombination, and carrier tunnelling from one molecule to another. The effective mobility may well be controlled by intermolecular transitions, not by intramolecular carrier motion. The trapping of charge, the percolation in charge transport and the effects of other localized charges will all have consequences which must be approached at the mesoscopic level. The several physical processes might be modelled in a number of possible ways. Our approach describes just one of these possible mesoscopic descriptions as an illustration of this class of methods.

\section{Components of a mesoscopic model}

For conventional crystalline semiconductors, like the III-Vs, optical properties are controlled by bandgap engineering: alloying so as to adjust the band edges without major compromises in lattice parameter. Many devices can be described successfully in terms of an isotropic homogeneous continuum model, using a standard semiconductor picture for properties such as carrier density and mobility. For conventional amorphous semiconductors, like a-Si:H, the optical properties are controlled both by the composition and by the precise preparation method. The transport properties have the extra complications of localized carrier states and of variable range hopping. Yet devices can still be described in terms of a continuum model, usually of a isotropic and homogeneous semiconductor. In the continuum pictures of both crystalline and amorphous semiconductors, space charge continues to be important, and is described in most cases by the Poisson equation for a consistently chosen continuum charge density. In the continuum descriptions of neither normal crystalline nor normal amorphous semiconductors does the structure or topology on the atomic scale enter explicitly (except perhaps through a macroscopic symmetry, like hexagonal symmetry for wurtzite structures).

The situation for organic semiconductors is quite different. Consider a typical polymer, consisting of molecular strands, with some statistical distribution of lengths, side-chains, crosslinking, texture and overall density. Some of the important features are determined largely by the individual molecular strands (e.g., intra-molecular mobility; band edge positions; relative importance of radiative and non-radiative recombination). Other features (e.g., isotropy of the 
macroscopic mobility; propensity to charge trapping) depend strongly on the connectivity and texture (meaning here both the 'spaghetti structure' and the possible presence of inclusions or voids). There is considerable experimental evidence that the texture is important (Bloor et al 1975, Friend et al 1999, Gao et al 1999, Nguyen et al 2000, Shi et al 2000, Whitehead et al 2000). Still further features depend on electron transfer between one component and another: electrode-molecule electron transfer (injection) and molecule-molecule electron transfer (inter-molecular aspects of mobility. There is therefore considerable potential to improve the performance of a device based on semiconducting polymers. It is not trivial to do so, since the various factors are interdependent. Among the challenges are these.

Challenge I is at the molecular level: how can one identify and model a molecule with the right electronic properties? In the main part, we shall have in mind our earlier self-consistent molecular dynamics calculations (Wallace et al 1991a, b, Ramos et al 1994) and corresponding treatments of metal-organic interfaces (Ramos et al 1993, Stoneham et al 1993, Stoneham and Ramos 1993), although there are many other approaches.

Challenge II concerns the identification of an acceptable production process, including integration with other materials.

Challenge III is at the microstructural level: how best can the ensemble of polymer molecules be organized to optimize performance for some application? This challenge (see also Ramos et al 1994, Ramos and Stoneham 1998, 2000a, b) is the focus of our attention here. We shall show there is considerable scope for control and optimization, especially if the texture can be controlled within the film. Further, some of the trends are compromises between trapping, transport across the film, radiative recombination and non-radiative recombination, and they are not always intuitive. The problems are even more subtle when there are inclusions within the polymer film. Moreover, there are differences between steady-state and pulsed operation. Even relatively simple calculations are of value.

Challenge IV concerns optimizing the use of macroscopic units (films, blobs, wires) in some application, whether electroluminescence, screening, sensors, smart materials, solar panels etc. This challenge includes recognizing the effects of conducting electrodes on the electromagnetic behaviour of a film which may be only a fraction of an optical wavelength thick.

We have implied that the performance of a device is dominated by the processes within the organic semiconductor. This is not wholly true. Clearly, there will be factors like the Fermi levels of the injecting electrodes, and electromagnetic boundary conditions which constrain the electric field to be normal to the surface of a conductor. In fact, the electrons in the conducting electrodes can lead to more subtle yet important effects, such as non-radiative transitions from energy transfer from the excited organic strand to the metal (Hochfilzer et al 1998); this effect is most important close to the electrode, apparently falling off with distance $d$ as $d^{-3}$.
1. Molecular level:
2. Properties of
e.g., self-consistent
molecular dynamics

\section{$\rightarrow$ molecule versus}
length, charge, curvature; inter-molecular tunnelling; injection from electrode
3. Mesoscopic
4. Construct
structure: texture, $\rightarrow$ realizations of crosslinks network

5. Electromagnetic theory (effects of electrodes because the film is less than a wavelength thick, image interactions, ....).

\section{Property}

$\rightarrow$ predictions for each realization 


\section{Realizations of polymer structures}

Our first aim is to mimic realizations of the 'spaghetti structure' of semiconducting polymers with chosen statistical characteristics. For example, we might want a particular range of polymer strand lengths and orientations. The orientations might be chosen to be different in different regions of the device. We might wish to have a particular degree of chain branching and cross-linking. We may want to there to be voids or inclusions. All these realizations will have to satisfy certain constraints, such as the avoidance of one polymer strand by another. Having created these realizations, we may wish to refine the structure, for example, by relaxing the geometry using self-consistent molecular dynamics, or otherwise. In the calculations we report here, the relaxation using molecular dynamics is omitted, since we shall use very simple descriptions of the transport. The fuller relaxation process is practical if needed.

Given these realizations of the polymer system, we shall carry out computer experiments in which charged carriers are injected. These carriers can move along polymer strands, and transfer from one strand to another, according to relatively simple rules. Carrier motion occurs under the influence of applied electric fields, the fields of other carriers, and the image charge because of the conducting electrodes. The carriers may simply carry current, or be trapped, or recombine radiatively or non-radiatively. It is the way that the texture affects the competition between these different processes which is of interest.

We have developed several approaches to produce realizations of polymer networks. These approaches will be compared in a later publication. In the present paper, we shall describe results based on a simple but flexible approach, which gives structures which we describe as nematic, in that the molecules have some degree of average alignment (Ramos et al 1994, Ramos and Stoneham 1998, 2000a, b). We start by generating a lattice in the space between the electrodes. In the present case, this will provide a basis for a model polymer film of thickness $90 \mathrm{~nm}$ and with a surface area of $100 \mathrm{~nm}^{2}$. The lattice does not have to be regular, but could be a disordered array of points. Our present work adopts a regular lattice, with planes parallel to the electrodes, and with cyclic boundary conditions. The density of lattice points is chosen to ensure the right density of the final polymer network. At each point (except for points chosen to belong to a void region) is placed a straight polymer strand of random length. The number of repeat units in each chain not directly contacting an electrode is chosen with uniform probability in the range 2 to 20 . Those chains directly bonded to the electrodes were chosen to have 20 units, so as to diminish the polarization attraction between the electrodes and the injected charges (of either sign). Initially, the units all have the same orientation, normal to the electrodes. A small random rotation is then assigned to each one of them. Within each plane, typical interchain distances were $0.7-1.4 \mathrm{~nm}$. The minimum distance permitted between the longest (20 unit) chains in adjacent planes was $1 \mathrm{~nm}$.

\section{Exploiting realizations of the polymer network: working approximations}

We are well aware of the complexity and subtlety of any full description of a semiconducting polymer device. In our present discussion, we shall make no attempt to include all these subtleties, although we note that ongoing work of ours addresses some of the issues.

Clearly, the work function and electron affinity of a particular strand depends on its length and curvature, as well as on the chemical nature. In previous work (Ramos et al 1994) we have estimated the dependences using self-consistent molecular dynamics. The results of such calculations could be incorporated without difficulty in our approach, but this is not done in the present paper. The work function also depends on the charge of the strand. This is a large effect in most cases, and the practical solution is to ensure that there is no more than 
one electronic charge (e or h) on a strand. Again, more detailed results are available (Ramos et al 1994). The probability of injection of a carrier from an electrode will depend, in general, on the work function and electron affinity, as well as on other features of the precise polymer conformation. We shall usually regard injection as either possible or not possible, with some pre-chosen random probability. It seems possible that only a relatively small fraction of the contacts actually lead to effective light emission (Ramos et al 1994). It would be valuable to have experimental checks of this aspect, for instance by scanning probe microscopy.

Studies of individual molecules show that the conformation of the individual molecules and their dimerization (a measure of single- and double-bond character along the molecule) are sensitive to charge state and to factors which affect the charge distribution, such as image interactions (Ramos et al 1994). Moreover, any tunnelling even, whether injection or transfer of a carrier to another polymer strand, will involve dynamical changes in conformation and dimerization. Such changes are a significant aspect of the charge transfer, and are now being examined by full quantum methods (Ness $\mathrm{H}$ and Fisher $\mathrm{A}$, private communication).

The charge injection step can take several forms. Here we discuss the injection of a number of carriers at the same time; we shall consider sequences of randomly timed injection events in a later paper. Electrons are injected from the cathode, holes from the anode. At a real electrode, injection of electrons or holes occurs through molecules in contact with the electrode. The probability of injection into a specific molecular strand will depend on its electron affinity and also the precise nature of the contact with the electrode. Whilst these features can be modelled, we shall make simpler assumptions here, and assume that injection occurs at specified sites where polymer strands contact an electrode. Such places we shall describe as 'good electrical contacts'. We shall assume that a certain fraction of molecules at each electrode are bad contacts, and do not accept electrons or holes from the electrode. We make two different assumptions. One assumption is that both carriers are blocked at that molecule. The other assumption is that only one carrier species (either electrons or holes) is blocked at a certain fraction of interfacial molecules, with the other species freely transferred. Blocking both types of carrier leads to more trapped charge than blocking just one type to the same degree. With this exception, our results show very similar qualitative behaviours in the two cases, although one can imagine polymer structures for which this would not be so.

Perhaps the most important aspect in practice is the final radiative recombination step. Recombination can occur on a single strand (Ramos et al 1994 discusses the evolution of an injected hole and an injected electron to form an exciton) or by recombination involving an electron on one strand and a hole on an adjacent one. These mechanisms have interesting differences. In particular, the dipole moment for the transition may be along the strand for intra-chain recombination, whereas the dipole moment for inter-chain recombination between two parallel chains is likely to be normal to their axes. In our present work, we shall only discuss intra-chain recombination. Whether recombination is radiative or nonradiative can depend on many factors. We shall adopt a simple rule, following experimental data for polydiacetylene (Sixl 1984): chains shorter than some chosen length show radiative recombination; recombination in longer chains is non-radiative. There are various possible reasons for this (Stoneham 1991). When we change the proportion of these short chains, we do so at constant number of chains, i.e., there is also a small density change. From our calculations for different but related models, we do not believe this has any significant effect on the interpretation of the results. In the comparisons below, when varying the proportion of small chains, we have kept the total number of chains constant, rather than constant density, so more short chains mean a slightly lower density.

Some special features can be readily added separately. For instance, there is an electromagnetic boundary condition requiring electric fields to be normal to a conducting surface. 
This is of most importance when considering optical absorption or emission, since the photon electric field is normal to its wave vector, favouring light propagating parallel to the electrodes.

A critical factor in charge evolution after injection is the total applied field, which is the sum of (a) the externally applied external field, here $2 \times 10^{8} \mathrm{~V} \mathrm{~m}^{-1}$ (Burroughes et al 1990), (b) the field of other charges in transit or trapped within the polymer network and (c) the field due to electrode polarization (cyclic boundary conditions are imposed; we evaluate the electric field contribution from the nearest image of each charge in the film). We emphasize the point that all charges are treated explicitly, rather than through a continuum space-charge description (e.g., Blades and Walker 2000). Such descriptions, usually based on the Poisson equation, are effectively a spatially averaged version of our more general approach.

We have adopted a simple description of the charge distribution over an individual chain. In this description, the charge is regarded as placed at one of three sites: one of the ends of the chain, or at the centre of the chain. When charge is transferred from site A1 on chain A to chain $\mathrm{B}$, it will arrive at the site on $\mathrm{B}$ closest to $\mathrm{A} 1$. The charge is then assumed to move instantaneously (i.e., before the next timestep) to the site on chain B which is favoured by the total electric field at B: in effect, the charge injected at one end of an individual chain moves along the chain under the influence of the field. Rapid intrachain motion and sluggish interchain motion is typical of many conducting polymer systems. For example, soliton velocities along a chain might be of the order of the sound velocity (say $10^{3} \mathrm{~m} \mathrm{~s}^{-1}$ ), so that motion along a chain of $10 \mathrm{~nm}$ would take of the order of $10^{-11} \mathrm{~s}$. Inter-chain hopping might be expected to have a rate of the form (some overlap factor) $\times\left(\right.$ jump rate of order $10^{13} \mathrm{~s}^{-1} \exp (-\varepsilon / k T)$ ); for $\varepsilon$ of $0.25 \mathrm{eV}$, the second factor would correspond to an inter-chain hopping time of order $10^{-9} \mathrm{~s}$, which would become even longer if the overlap factor is small. Our results work entirely in timesteps, rather than real time. However, for guidance, one should think of the timestep as being of order $10 \mathrm{ps,} \mathrm{which} \mathrm{would} \mathrm{ensure} \mathrm{that} \mathrm{all} \mathrm{the} \mathrm{physically} \mathrm{relevant} \mathrm{times} \mathrm{are}$ of the right order and relative magnitude. We have results for carrier dynamics, but we shall defer discussion of them to a later paper. The distribution of carrier arrival times is in line with other predictions: a reasonably rapid rise after the first carriers can cross the film, a maximum corresponding to the most probably arrival time, and tail in the distribution from those carriers which took long routes or were trapped transiently. We are aware of experimental information (e.g., Giebeler et al 1999).

The transfer of carriers between chains is crucial, since the organic film thickness is typically tens or hundreds of molecular lengths. For the present, we shall use the following simple rules for interchain transitions. First, the electron or hole will jump to the nearest neighbour chain for which it has the greatest hopping probability. Thus a charge injected at one electrode percolates through the polymer film, making a series of jumps to those neighbour chains with the greatest hopping probability $w /\left(\sum w_{i}\right)$; the sum here is over all the nearest neighbour strands and $w$ is the jump rate. Secondly, the jump rate is assumed to be the product of two factors. One factor falls off exponentially with the distance between the centres of the two molecules involved (Ramos and Stoneham 2000a). The other factor modulates the probability by favouring transitions in the direction favoured by the electric field. We are actively developing more accurate quantum descriptions, but these simple rules shows many of the significant features. Indeed, since we are simply selecting one most probable jump, and we know the closest proximity and maximal field assistance are crucial, many variants of the precise algorithm should give essentially the same behaviour. Thirdly, we shall not allow more than one electron, or more than one hole, to be present on any molecule. No two carriers of the same sign are allowed on a chain. It is straightforward to generalize our approach to include the more complex effects of the electric field on jump and recombination probabilities, but this is not done in the present calculations. 
An important feature is that the carrier can be trapped on a chain, even when the chain has an electron affinity similar to the other chains. Of course, such a trapped charge still contributes to the space charge. We shall describe a carrier as trapped if (a) the total electrical field on the charge is zero, (b) the carrier meets a cross-link and also the electric field is less than a certain critical value or (c) the jump probability per timestep to a neighbouring chain $(w)$ is less than $10^{-5}$. If we recall that a timestep is likely to be of the order of $10^{-11} \mathrm{~s}$, then this value of $w$ would correspond to a rate of less than one-thousandth of the typical inter-chain hopping rate at room temperature, i.e., they remain would remain trapped for more than 1000 timesteps. Since almost all the carriers which traverse the polymer from one electrode to the other do so in a few tens of timesteps, the charge is indeed properly trapped as regards its influence on conduction. Whether it is trapped well enough to permit standard measurements of space charge is less clear. The jump probability will depend on temperature, and the specific limit we take could also be regarded as defining a temperature. Clearly, a particular carrier may be trapped at one time and not trapped after some other charges have moved and the electric field has changed, or carriers have moved away from those strands adjacent to the trapped carrier. We emphasize that our model can show trapping even when there are no impurities or structural defects (as normally defined). Trapping can occur because of the topology (linkage) of the polymer structure, combined with the effect of the total local field. This type of space charge does not exist in a homogeneous, defect-free, crystalline semiconductor. Clearly, some of the analogies with such standard semiconductors as Si can only be used for polymeric semiconductors with considerable caution.

As noted already, recombination occurs during the course of the simulation when two oppositely charge carriers meet on the same chain. This recombination can give rise to luminescence, or it may be non-radiative, giving heat, depending on factors such as chain length. We shall show some results for polydiacetylene where, following Sixl (1984), we assume that the longer polydiacetylene chains, those with more than eight units, do not luminesce. We shall not discuss excitonic or excitation transfer processes here.

\section{Results: competition between processes}

We are interested in four main classes of process. First, there is conduction across the polymer, which is measured by the fraction of carriers reaching target electrode. Conduction is undesirable in light-emitting diodes, since the carriers do not contribute to the light output and do generate Joule heating. Secondly, there is trapped charge. Such trapped carriers might recombine at some later stage, but they are again wasted carriers which add to the unpredictability of device operation. They may, for example, lead to areas of the device which are ineffective. Thirdly, there is non-radiative recombination. Recombination is measured by the number of recombination events per $\mathrm{e}-\mathrm{h}$ pair injected. Like conduction, non-radiative processes generate heat instead of light. Finally, there is radiative recombination. Where within the device this radiative recombination occurs may be important for the overall efficiency, partly because of the electromagnetic boundary conditions.

Our results here concentrate on three factors: the effects of varying the proportion of short chains; the effects of varying the numbers of 'good' contacts and the effects of inert spherical inclusions. Some of the results are natural. For example, it is obvious that building in nonconducting inclusions will reduce the current efficiency (the fraction of carriers which complete a journey from one electrode to the other), although it is less clear how much conduction will be reduced. However, the competition between the several key processes of carrying current, trapping and radiative or non-radiative recombination can lead to results which are by no means intuitive. One reason for such unexpected results is that altering one feature affects several 
$100 \%$ injection efficiency

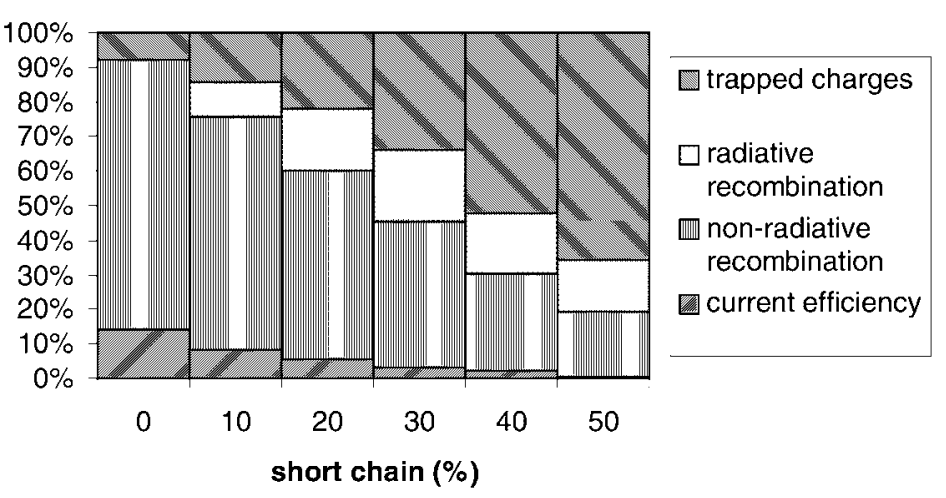

(a)

$70 \%$ injection efficiency

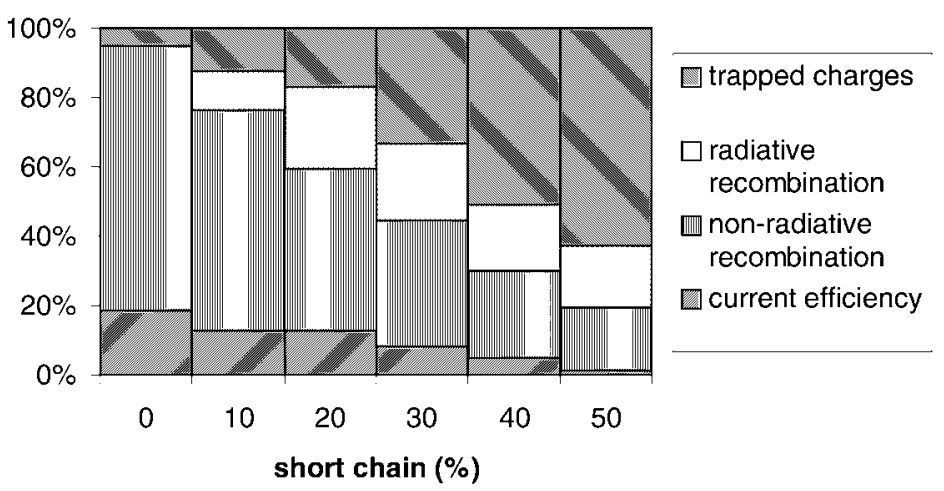

(b)

$40 \%$ injection efficiency

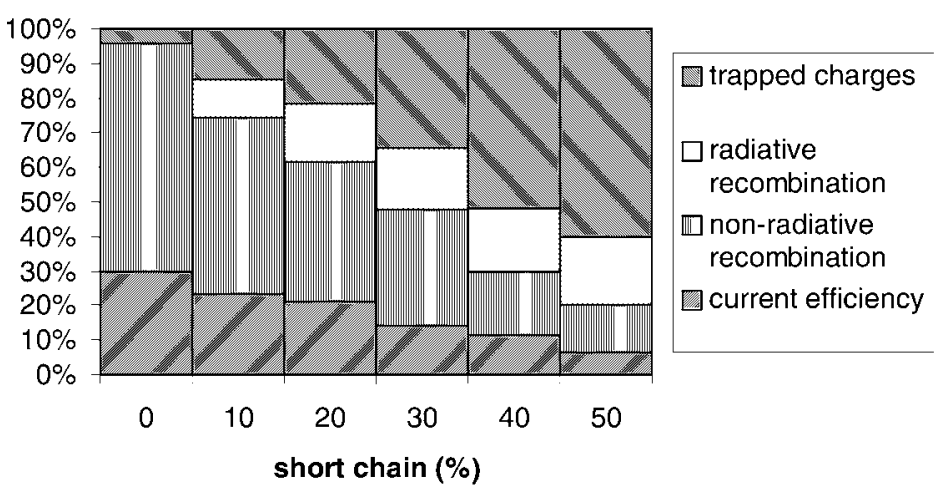

(c)

Figure 1. Proportions of injected charge which are trapped, recombine radiatively or nonradiatively or which cross the film to carry a current, as a function of the fraction of shorter chains. The histograms show broadly similar results for different injection efficiencies: (a) 100\%; (b) $70 \%$; (c) $40 \%$ good electrode contacts. 

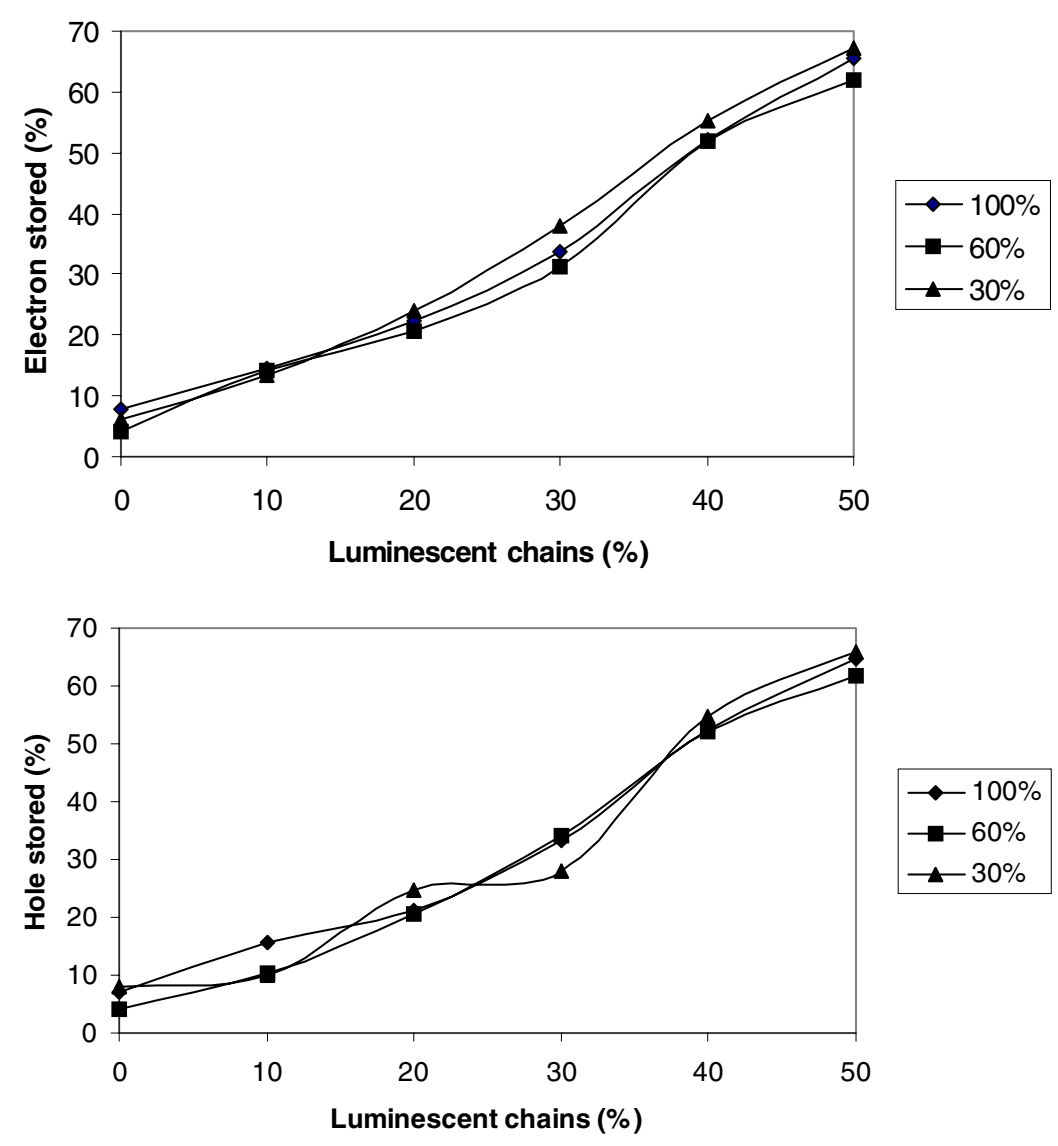

Figure 2. As the proportion of shorter (luminescent) chains rises, trapping increases. The results are largely independent of the proportion of good electrode contacts (100\%: diamonds; $60 \%$ : squares; $30 \%$ : triangles). The lines are a guide to the eye.

aspects of behaviour. For example, putting in more short chains means that there are more chains on which radiative recombination can occur, but it also alters the topology and affects trapping and conduction.

\subsection{Trends with increasing numbers of short chains}

We have noted that, at least for certain polymer systems, it is only the shortest chains which luminesce. We can change the relative numbers of shorter (luminescent) and longer chains. The results show several general trends, irrespective of the proportions of good contacts. These are summarized as histograms in figure 1.

The first significant trend is increasing trapping (figure 2). An initial analysis of where charge is trapped suggests that most of the trapped charge is near the middle of the film, with electrons nearer the cathode and holes nearer the anode (this is modified when there are inclusions; see section 5.3). A number of the local charge distributions suggest that trapped charges are pinning other charges in sites which are unfavourable for jumps along the local field, as one might expect. The second clear trend is a decrease in current efficiency. Current efficiency and trapping anticorrelate (figure 3). 


\section{$100 \%$ injection efficiency}

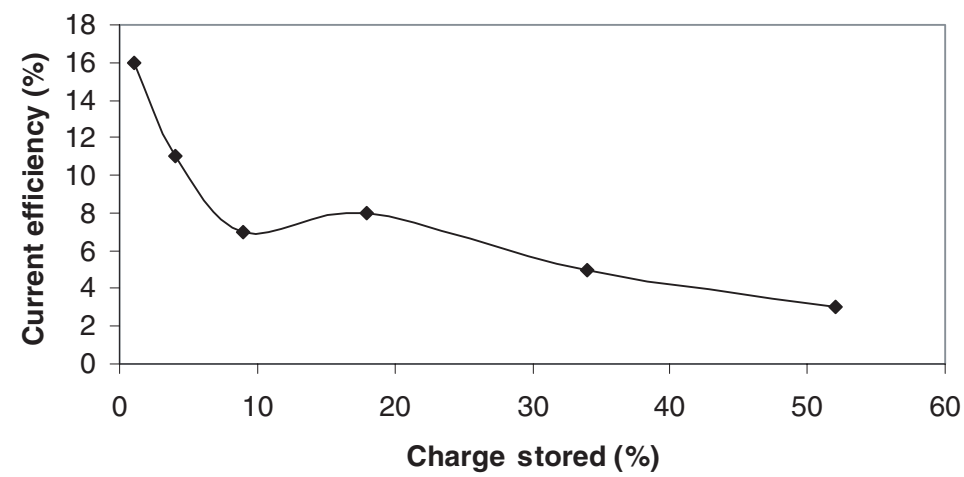

Figure 3. Current efficiency (the proportion of carriers crossing the film to reach the other electrode) anticorrelates with trapping, even when there is significant recombination. The line is a guide to the eye.

A third trend is a decrease in total and non-radiative recombination when there are more short chains, whereas (as expected) radiative recombination increases for the first few short chains. What is unexpected is that luminescent recombination saturates when there are about $20 \%$ of short chains. Increasing the number of luminescent chains does not always lead to increases in optical efficiency (figure 4). There may even be a slight decline in radiative recombination when the fraction of short chains is above about $35 \%$. This result can also be seen in another way (figure 5), in which we look at the trends in recombination efficiency with charge stored, the number of shorter chains being a hidden parameter, varying along the curve. We can also examine the spatial distribution of the recombination events. For the structures considered here, both radiative and non-radiative recombinations occur primarily in the centre of the film, as one might expect. We shall report separately results for other polymer mesostructures, notably those with regions where there are chains (statistically) normal to the electrodes and regions where there are chains (statistically) parallel to the electrodes. In such cases, the spatial distribution of recombination can be very different.

\subsection{Trends with reducing injection efficiency}

The injection efficiency can be varied by adjusting the fraction of good molecule to electrode contacts. Again, there are general trends, holding for all proportions of short (luminescent) chains; these can be seen from the histograms (figures 1(a), 1(b), 1(c)).

The first trend is that trapping increases with reducing injection efficiency. This seems natural, since some of the injected carriers will be unable to leave at the opposite electrode. However, there is a second, less obvious, trend, namely increased current efficiency. Together, the trends are not intuitive. A third trend with decreasing injection efficiency is a decrease in total recombination and in non-radiative recombination. Radiative recombination remains roughly constant until there are less than about $20 \%$ good contacts, when the radiative recombination also decreases somewhat. 

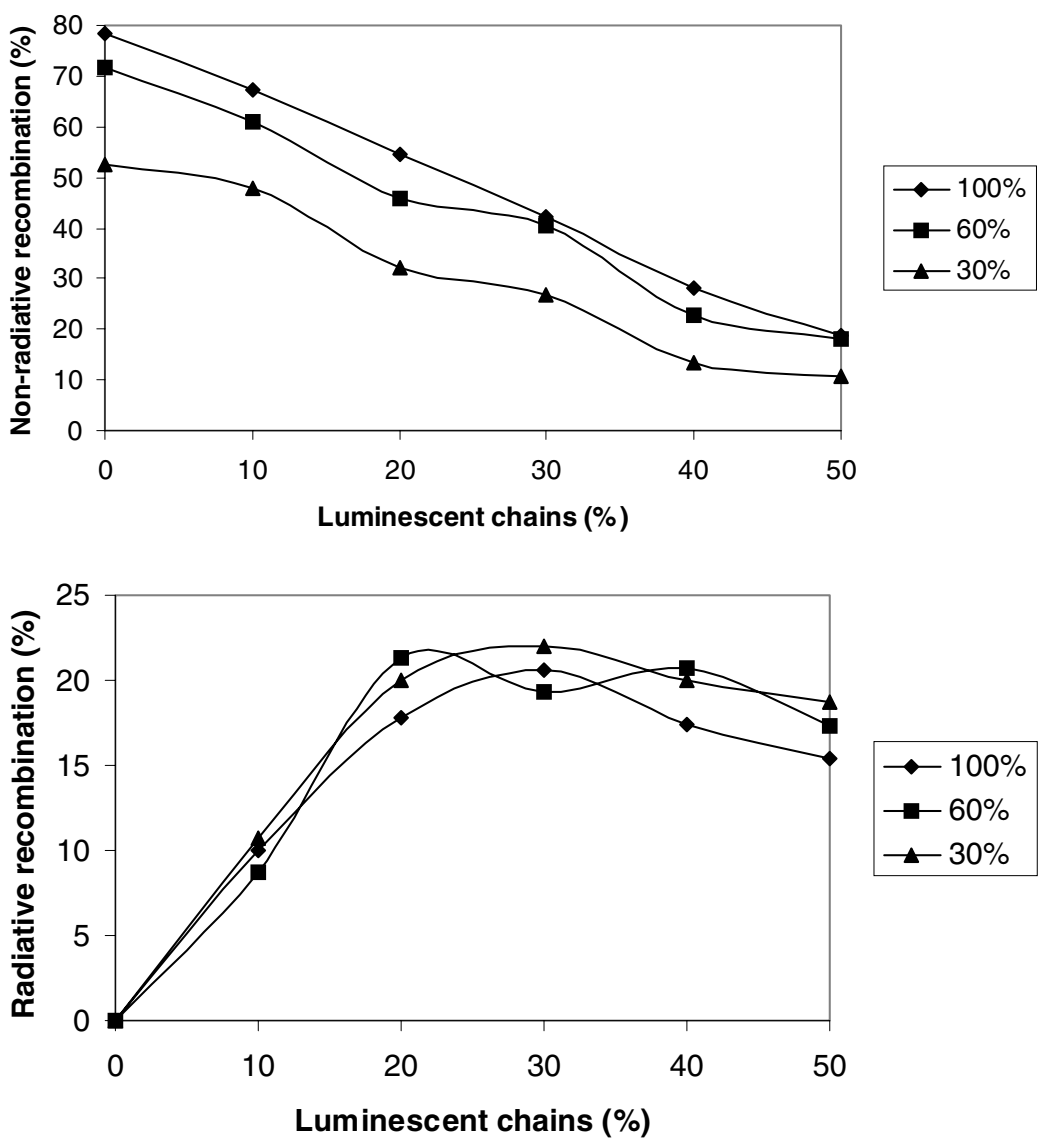

Figure 4. As the proportion of radiative (shorter) chains increases, non-radiative recombinations decrease (as expected), but radiative recombination rises and then saturates, as trapping becomes more important. Saturation suggests a limit on luminescence efficiency. The lines are a guide to the eye.

\subsection{Trends with inclusion density}

An inclusion is a sphere of inert material, here taken to have a diameter of $2.1 \mathrm{~nm}$. The inclusion excludes polymeric material from the spherical volume, and we shall define to inclusion concentration by the percentage of polymer strands removed in consequence; we have used fractions excluded up to $20 \%$. In our present work, the sphere neither attracts nor repels carriers. A sphere of different dielectric constant will affect carriers through the image interaction, and we shall report the effects of such weak traps in a future paper. There are many studies of mixed systems of conducting polymers with small particles of other materials, usually inorganic (e.g., Daubler et al 1999, Salafsky 1999, Lee et al 2000, Wu Guozhang et al 2000).

Again, there are some general trends, irrespective of the short chain fraction or the injection efficiency (figure 6). We are able to check our statistics by confirming similar results for electron and hole currents. There is evidence of blocking by the inclusions, as expected. Trapping increases with the number of inclusions, presumably because there are more 'dead end' paths. There is more trapped charge close to the electrodes than in the absence of inclusions, as one would expect if paths were blocked. Total recombination falls roughly linearly with the 


\section{$100 \%$ injection efficiency}

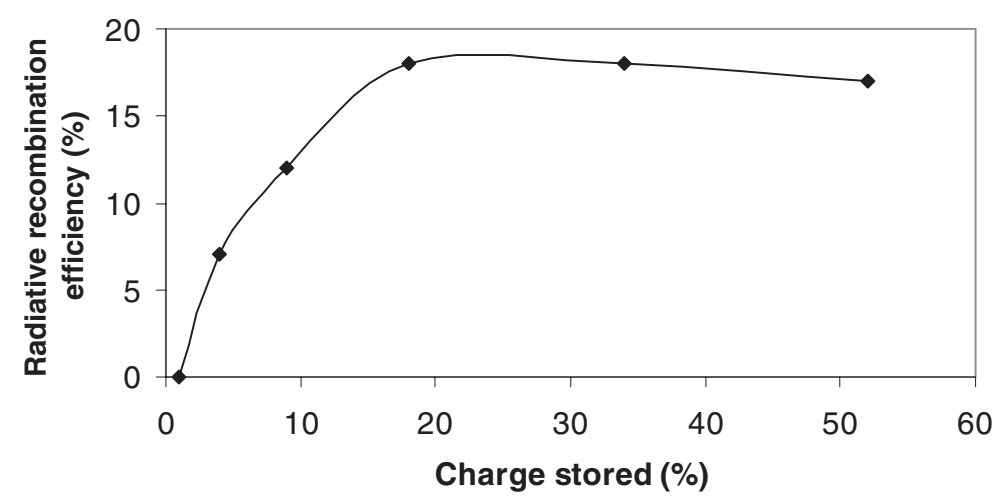

$100 \%$ injection efficiency

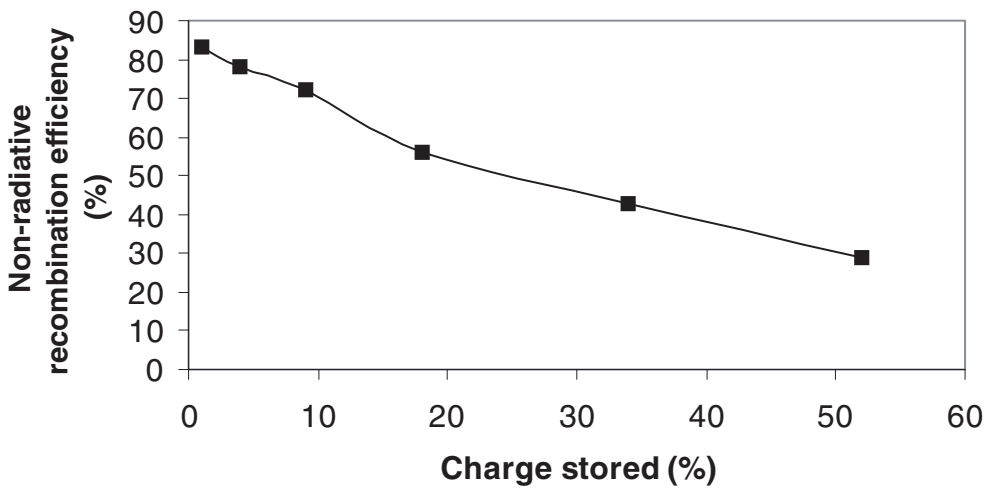

Figure 5. Trends in recombination with charge stored by trapping. There is an implicit change in the proportion of shorter chains along the curves. Note the monotonic trend for non-radiative transitions, in contrast to the saturation of radiative recombination. The lines are a guide to the eye.

number of inclusions, but perhaps more rapidly than one might have guessed: with $50 \%$ short chains, for instance, increasing the inclusions from zero to $20 \%$ reduces the recombination from $35 \%$ to $5 \%$. Non-radiative recombination changes similarly to total recombination. Radiative recombination shows some wide statistical variations, apparently peaking at about 20-30\% short chains in most cases.

\section{Conclusions}

The interplay of length scales is evident in many systems. Our mesoscopic modelling provides one means to link atomistic studies of individual molecules to the continuum descriptions common in device design. The specific realizations and computer experiments described show several important features. One is the existence of 'trapping', even in a model in which there are no defect or impurity states; in such trapping, carriers may be localized for times 
$0 \%$ short chain

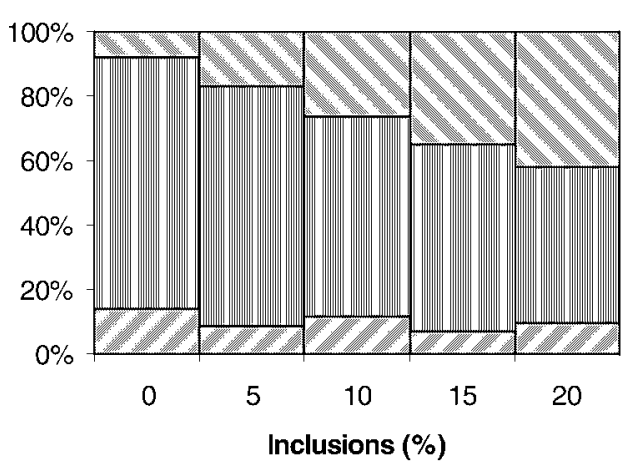

trapped charges

$\square$ radiative recombination

ill non-radiative recombination

国 current efficiency

(a)

$20 \%$ short chain

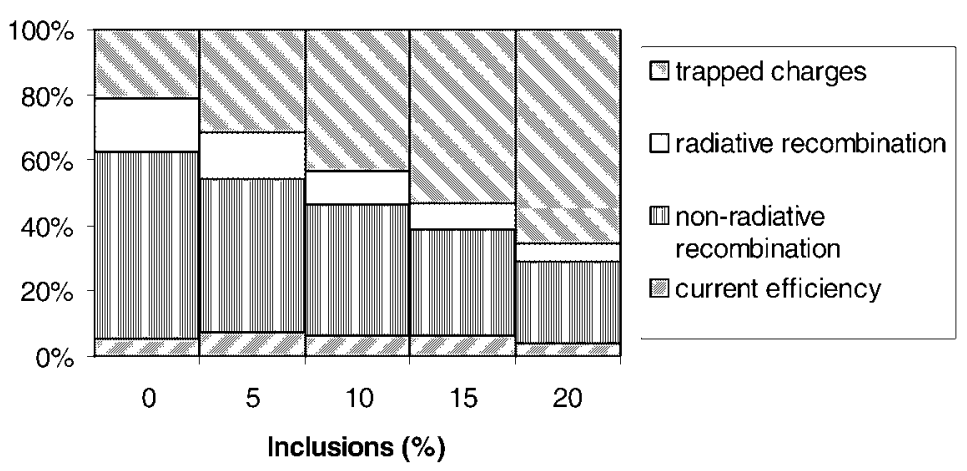

(b)

$40 \%$ short chain

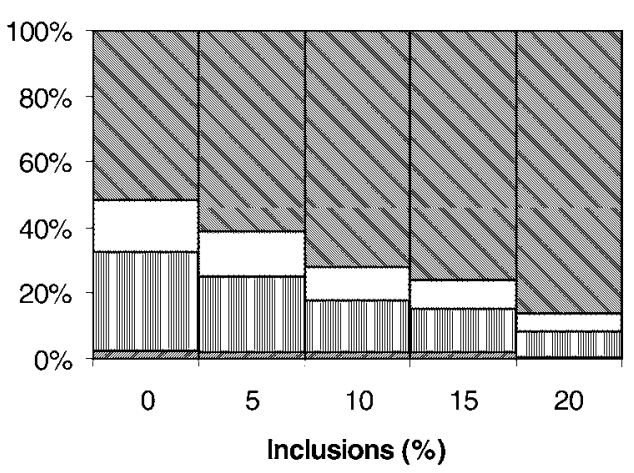

trapped charges

$\square$ radiative recombination

四 non-radiative

recombination

圈 current efficiency

(c)

Figure 6. The effects of inert inclusions, shown for (a) $0 \%$, (b) $20 \%$, (c) $40 \%$ short chains. The effects of increasing inclusions and of increasing shorter chains are both to increase trapping. 
long compared with the typical transit time of those carriers which traverse the film. The fact that there is competition between trapping, transport and recombination (radiative and nonradiative) leads to systematic trends, some being obvious, others being non-intuitive, as one varies factors such as the proportions of short luminescent chains, of good electrode contacts or of inert inclusions. One striking result is the saturation of luminescent efficiency at a modest proportion of shorter chains.

Perhaps more important is simply the fact that modelling is possible, and is very flexible. We shall be reporting separately results for a range of textures, for example, and we are able to include the several effects associated with the presence of materials with different dielectric properties in a device. We believe the approach could provide a useful design tool for applications ranging from electrode design and texture optimization for applications ranging from light emission to solar panel design. It further seems likely that the approach may be useful in studies of electrical breakdown in insulating polymers (Stoneham 1998).

\section{Acknowledgments}

We are indebted to Dr Alison Walker, Dr Andrew Fisher and Dr Hervé Ness for discussions. We should also like to express our thanks to Professor William Hayes for introducing us to conducting polymers and for his continuing support and encouragement. Some of this work was supported by the EPSRC.

\section{References}

Blades C D J and Walker A B 2000 Synth. Met. 111 335-40

Bloor D, Koski D, Stevens L, Preston G C and Ando F H 1975 J. Mater. Sci. 101678

Burroughes J H, Bradley D C, Brown A R, Marks R N, Mackay K, Friend R H, Burns P L and Holmes A B 1990 Nature 347539

Daubler T K, Glowacki I, Scherf U, Ulanski J, Horhold H H and Neher D 1999 J. Appl. Phys. 866915

Friend R H et al 1999 Nature 397121

Gao Z Q, Lai W Y, Wong T C, Lee C S, Bello I and Lee S T 1999 Appl. Phys. Lett. 743269

Giebeler C, Whitelegg S A, Campbell A J, Liess M, Martin S J, Lane P A, Bradley D D C, Webster G and Burn P L 1999 Appl. Phys. Lett. 743714

Hochfilzer C, Leising C, Gao Y, Forsythe E and Tang C W 1998 Appl. Phys. Lett. 732254

Lee J, Sundar V C, Heine J R, Bawendi M G and Jensen K F 2000 Adv. Mater. 121102

Nguyen T Q, Kwong R C, Thompson M E and Schwartz B J 2000 Appl. Phys. Lett. 762454

Ramos M M D and Stoneham A M 1998 Proc. 3rd Int. Conf. on Electric Charge in Solid Insulators (1998) Supplément à la Revue 'Le Vide: Science, Technique et Applications' No 287, ed G Damamme, p 5

-2000a Comp. Mater. Sci. 17260

2000 b Synth. Met. at press

Ramos M M D, Stoneham A M and Sutton A P 1993 Acta. Metall. Mater. 41 2105-11

-1994 Synth. Met. 67137

Salafsky J S 1999 Phys. Rev. B 5910885

Shi Y, Liu J and Yang Y 2000 J. Appl. Phys. 874254

Sixl H 1984 Polydiacetylene ed D Bloor (Amsterdam: Nijhoff) pp 240-5

Stoneham A M 1991 Optical Properties of Excited States in Solids ed B di Bartolo (New York: Plenum)

—1998 Proc. 3rd Int. Conf. on Electric Charge in Solid Insulators (1998) Supplément à la Revue 'Le Vide: Science, Technique et Applications' No 287, ed G Damamme, p 1

Stoneham A M and Ramos M M D 1993 J. Solid State Chem. 106 2-12

Stoneham A M, Ramos M M D and Sutton A P 1993 Phil. Mag. A 67 797-811

Wallace D S, Stoneham A M, Hayes W, Fisher A J and Harker A H 1991a J. Phys.: Condens. Matter 3 3879-904

Wallace D S, Stoneham A M, Hayes W, Fisher A J and Testa A 1991b J. Phys.: Condens. Matter 3 3905-20

Whitehead K S, Grell M, Bradley D D C, Jandke M and Strohriegl P 2000 Appl. Phys. Lett. 762946

Wu Guozhang et al 2000 J. Appl. Phys. 881480 